Journal of Accident and Emergency Medicine 1994

11, 213-217

Correspondence: Wong Tai-wai, Consultant, Accident and Emergency Department, Kwong Wah Hospital, 25 Waterloo Road, Yaumati, KIn. Hong Kong

\title{
Reattendance audit in an inner-city emergency department
}

\author{
T.-W.WONG \& K.-W. LAM
}

Accident and Emergency Department, Kwong Wah Hospital, 25 Waterloo Road, Yaumati, Hong Kong

\section{SUMMARY}

A study was carried out in an inner-city emergency department (ED) of patients returning for related complaints within $48 \mathrm{~h}$ of discharge. The incidence was around $0.7 \%$ and the majority $(82 \%)$ of patients presented with persistence or progression of the original symptoms. Patient-related factors accounted for $13 \%$ of returns and only $5 \%$ were found to be the result of doctor-related factors. Of the returnees, $54 \%$ were discharged and $36 \%$ were admitted. The most common initial complaint was pain (31\%) which, in $23 \%$ of patients, was localized to the abdomen. Injury (14\%) and febrile illnesses (13\%) came second and third. Asthma and chronic obstructive airway diseases (COAD) also accounted for $8 \%$ of cases. Children of less than 10 years of age were more likely to return within $48 \mathrm{~h}$. About $18 \%$ of cases were thought to be potentially avoidable. Better patient education and an improvment in primary health care services were thought to be important in decreasing such avoidable reattendance. Reattendance audit was a useful tool in the continuous quality improvement programme of emergency department.

Key words: audit, quality assurance, reattendance, unscheduled revisit

\section{INTRODUCTION}

Emergency departments (EDs) are seeing more and more patients nowadays. Nearly all patients are new to us and it is thus very important to make sure they are assessed carefully and discharged safely from the department. It is necessary to have some mechanism to monitor discharges. Pierce ${ }^{1}$ has shown that daily chart review did not help to detect most of the avoidable returns. Reattendance audit is also suggested as part of the quality assurance programme for EDs. ${ }^{2}$

In this study we explore the characteristics of patients who came back to the department within $48 \mathrm{~h}$ and the reasons for their return visits. We would also like to identify high risk groups for return visits and devise possible strategies to decrease avoidable return visits.

\section{STUDY SETTING}

Kwong Wah Hospital is a general hospital situated in the city centre of Hong Kong. It is a public hospital under the Hospital Authority and it has about 1500 beds. The ED had over 130000 attendances per year during the study period. The department was staffed with one consultant, four senior medical officers and 13 medical officers. The department provides a 24-h service to patients who have a wide spectrum of acute complaints. About $20 \%$ of patients were brought in by ambulance and the rest were walk-in patients.

\section{PATIENTS AND METHODS}

All patients who returned to the ED within $48 \mathrm{~h}$ during the 8-month study period from 1 September 1991 to 30 April 1992 were included. At the time of study, patient registration data were not computerized and therefore we relied on the patient to give us a history of recent visits. The records of the patients were reviewed by the first author. The patients' age, sex, presenting complaints on the two occasions, the reasons for the return as well as the outcome of the second visit were recorded and fed into a dBase IV programme for analysis. The reasons for return were ascertained from the records and were classified by the author as being the result of factors pertaining to the illness, the patient, the doctor or the system.

\section{RESULTS}

The total attendance of new patients during the 
T.-W. Wong \&

K.-W. Lam study period was 90154 and the number of nonscheduled return visits was 658 of which 42 were judged to be because of unrelated problems. The rate of non-scheduled returns within $48 \mathrm{~h}$ for the population was around $0.7 \%$. We concentrated our analysis on those 616 patients who came back with related problems.

The age distribution is shown in Figure 1. The age distribution of all patients attending the ED was studied in the week from 1-7 December 1991 and is also shown in Fig. 1 for comparison. Of the 616 returnees $353(57 \%)$ were male and $263(43 \%)$ were female.

The most frequent reason for returning to the ED was persistence or progression of the illness that had brought the patient to the department in the first place (Table 1). Complications of illness, e.g. wound infection, accounted for only $3 \%$ of returns.

Patient-related factors were the second most important and accounted for $13 \%$ of reattendances (Fig. 2). The two most frequently occurring factors in this group were patients who had discharged themselves against medical advice $(50 \%)$ and patients who returned for sick leave certificates $(30 \%)$. A small proportion $(7.5 \%)$ of patients had actually disappeared without trace in the first visit.

Doctor-related factors only accounted for $5 \%$ of patients who returned unscheduled (Table 2). Untoward reactions to drugs were the cause for return in nearly half of the patients within this group.
Table 1. Reasons for return visits

\begin{tabular}{lcc}
\hline $\begin{array}{l}\text { Reason for } \\
\text { return }\end{array}$ & $\begin{array}{c}\text { No. of } \\
\text { patients } \\
\text { returning (\%) }\end{array}$ & $\begin{array}{c}\text { No. of } \\
\text { patients } \\
\text { admitted (\%) }\end{array}$ \\
\hline $\begin{array}{c}\text { IIness- } \\
\text { related } \\
\text { Patient- } \\
\text { related }\end{array}$ & $504(82)$ & $179(36)$ \\
$\begin{array}{c}\text { Doctor- } \\
\text { related } \\
\text { System- } \\
\text { related }\end{array}$ & $80(13)$ & $32(40)$ \\
\hline
\end{tabular}

Table 2. Breakdown of doctor-related reasons for reattendance

\begin{tabular}{lc}
\hline Doctor related & No. of patients (\%) \\
\hline Treatment error & $17(57)$ \\
Drug reaction & $13(40)$ \\
No analgesics & $3(10)$ \\
Inadequate & $2(7)$ \\
Misdiagnosis & $8(27)$ \\
Abdominal pain & $4(13)$ \\
Injury & $2(7)$ \\
Dizziness & $1(3)$ \\
Fever & $1(3)$ \\
Failure to refer & $4(13)$ \\
Failure to admit & $1(3)$ \\
\hline
\end{tabular}

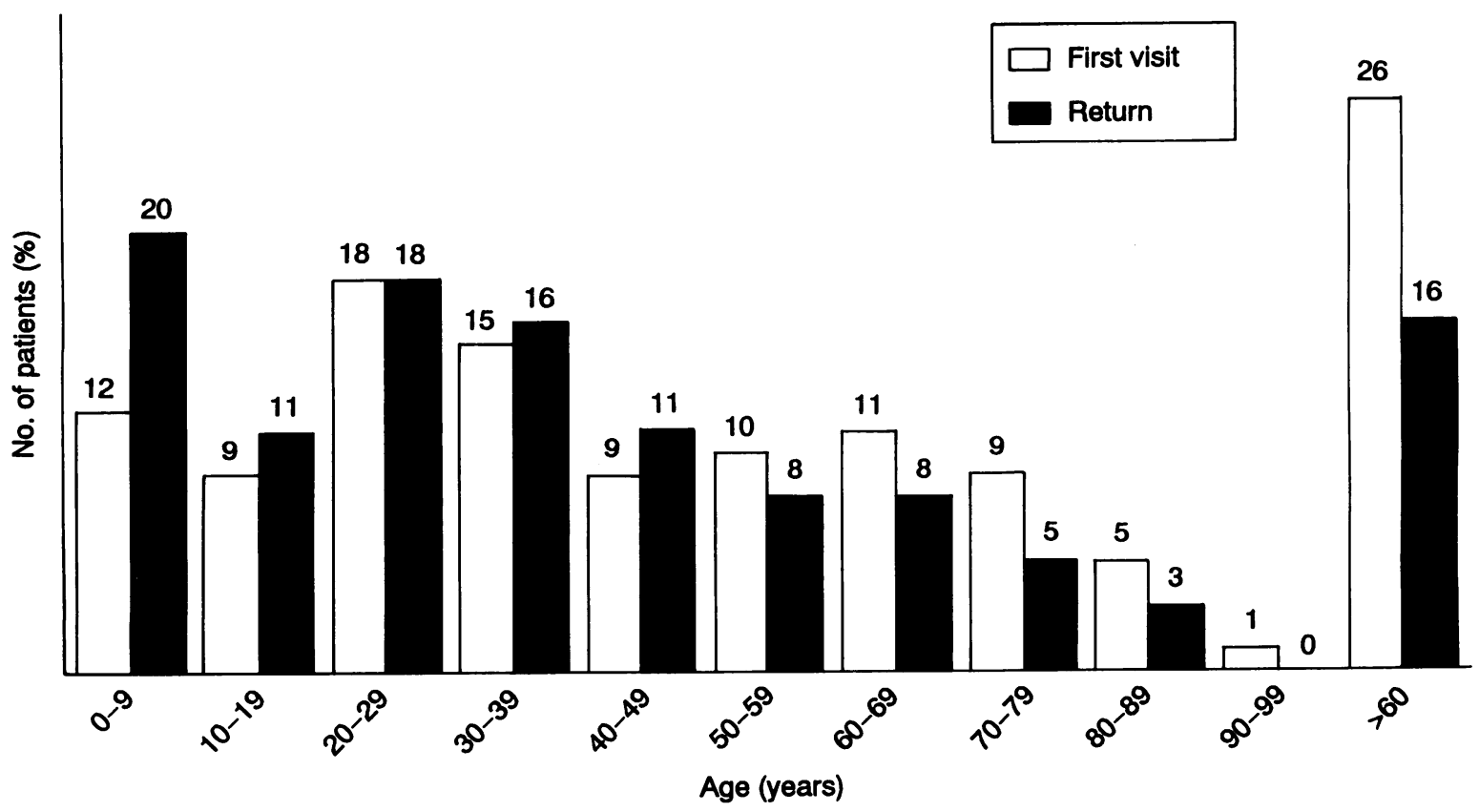

Fig. 1. Age distribution of patients. 
Reattendance audit

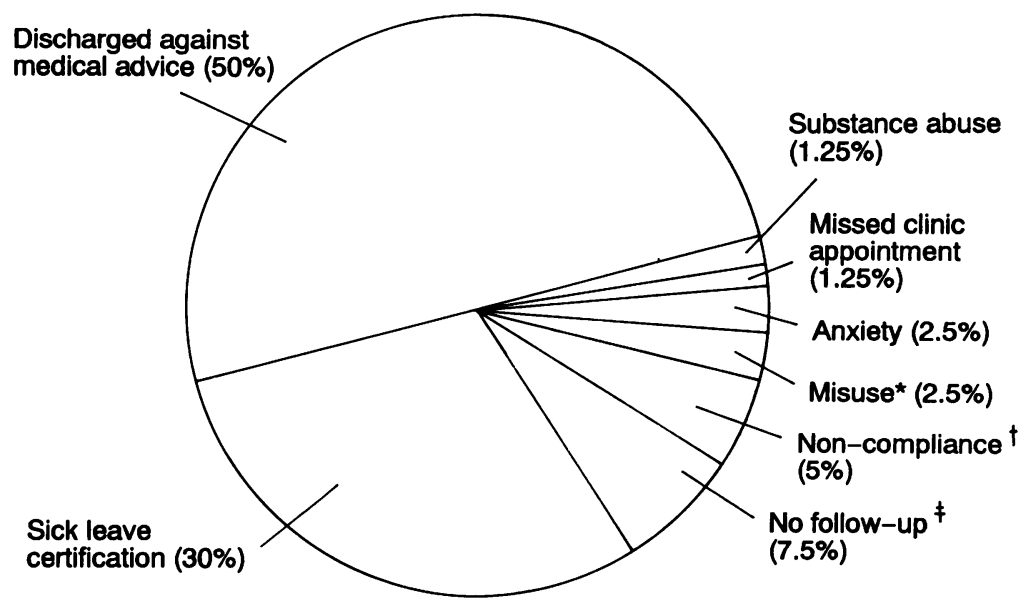

Fig. 2. Patient related reasons for reattendance.

Misdiagnosis was second in this group and accounted for $27 \%$ of cases. The rest reattended because they had not been prescribed analgesics or had not been admitted or referred on the first visit. System factors, which include for example, unavailablity of an early specialist out-patient appointment or closure of the pharmacy, were negligible in this study.

Most of the reattenders $(54 \%)$ were discharged after the second consultation (Table 3 ). A substantial proportion $(36 \%)$ were admitted and among the patients admitted nearly $90 \%$ had persistence or progression of the original illness. Only $5 \%$ of reattenders were admitted because of doctor's mismanagement. About $5 \%$ of reattenders were referred to specialist clinics and $4 \%$ discharged themselves against medical advice. There was one patient who was certified dead on return to the department presumably as a result of acute myocardial infarction which was not diagnosed at the first visit.

The most frequently presenting symptom in the first visit was pain. Painful conditions accounted for $188(31 \%)$ reattenders of whom $135(23 \%)$ presented with some form of abdominal pain. Injuries and febrile illness were second and third most com-

Table 3. Outcome of return visit

\begin{tabular}{lc}
\hline Patient outcome & No. of patients (\%) \\
\hline Discharged & $332(53.9)$ \\
Admitted & $222(36.0)$ \\
Referred & $33(5.4)$ \\
Self-discharge against & \\
$\quad$ medical advice & $25(40.1)$ \\
Disappeared & $3(0.5)$ \\
Died & $1(0.2)$ \\
\hline
\end{tabular}

mon symptoms and together accounted for $27 \%$ of the patients. Most of the patients with a complaint of fever were children of less than 10 years of age. Gastroenteritis and obstructive airway diseases accounted for about $20 \%$ of patients. Of those presenting with obstructive airways diseases (asthma and COAD) over half were admitted in the second visit. About $\mathbf{4 0} \%$ of patients with initial complaints of pain or fever were also admitted. In the injury group, 16 patients $(19 \%)$ were admitted who were mainly suffering from head injury (Table 4).

\section{DISCUSSION}

The incidence of $0.7 \%$ of patients making an unscheduled return visit in our study was higher than that reported by Lerman ${ }^{3}$ but was lower than most other studies ${ }^{1,4}$ reported in North America. One obvious reason was the different time frames used in the studies (Table 5). We were using $48 \mathrm{~h}$ as our

Table 4. Presenting symptoms of return visit patients

\begin{tabular}{lc}
$\begin{array}{l}\text { Presenting } \\
\text { symptoms }\end{array}$ & No. of patients (\%) \\
\hline Painful conditions & $188(31)$ \\
Abdominal pain & $139(23)$ \\
Musculoskeletal & $24(4)$ \\
Renal colic & $6(1)$ \\
Others & $19(3)$ \\
Injury & $84(14)$ \\
Febrile illness & $79(13)$ \\
Gastroenteritis & $61(10)$ \\
Asthma/COAD & $49(8)$ \\
URI & $31(5)$ \\
Skin condition & $22(4)$ \\
Dizziness & $14(2)$ \\
Others & $88(14)$ \\
\hline
\end{tabular}




\begin{tabular}{llllll}
\hline & \multicolumn{5}{c}{ Author } \\
\cline { 2 - 6 } Statistics & Wong & $\mathrm{Hu}$ & Keith & Pierce & Lerman \\
\hline Time & $48 \mathrm{~h}$ & 7 days & $72 \mathrm{~h}$ & $48 \mathrm{~h}$ & $72 \mathrm{~h}$ \\
$\begin{array}{l}\text { Reattendance } \\
\text { Avoidable } \\
\quad \text { return }\end{array}$ & $0.7 \%$ & $4.9 \%$ & $3.4 \%$ & $3.3 \%$ & $0.4 \%$ \\
\begin{tabular}{l} 
Case finding \\
\hline
\end{tabular} & $18 \%$ & $8 \%$ & $32 \%$ & - & $32.6 \%$ \\
\hline
\end{tabular}

Table 5. Reattendance statistics of different studies inclusion criteria whereas some other studies used $72 \mathrm{~h}$. The other factor was the use of computers in the identification of return visits. In our study, as well as in Lerman's study, ${ }^{3}$ case finding was carried out manually based upon reports from patients and many cases could be missed if patients did not volunteer a history of a previous visit. As with other studies, we could not be certain of the incidence of patients returning to a different facility for further care. In a study ${ }^{5}$ by the Department of Community Medicine of the University of Hong Kong over $20 \%$ of patients sought further treatment after the initial visit but only $2 \%$ opted to go back to the ED for further consultation.

We chose a 48-h time frame as this had been found to be cost effective in a previous study. ${ }^{4}$ In our experience patients in Hong Kong were impatient and they would certainly seek further medical opinion if their symptoms were not cured in a day or two. The official management information statistics of the Hospital Authority which used 72-h return as the inclusion criteria did yield an incidence similar to the present study. Thus, it was probably adequate to use $48 \mathrm{~h}$ as the cut off point in our setting.

In our study the majority of return visits were illness-related and this was similar to a study in a New Zealand ED. ${ }^{6}$ The results of Pierce, ${ }^{1}$ however, were different, reporting patient related factors as the most important cause of reattendance. This might be the result of a difference in the organization of health care. In Hong Kong most people do not have a family physician and they would attend government out-patient clinics or local EDs for episodic illness. Since EDs are convenient with shorter waiting times and also free of charges, many citizens use EDs for primary care problems. As many as $61 \%$ of respondents said they would choose an ED for routine primary care in a study ${ }^{5}$ conducted by the Community Medicine Department of the University of Hong Kong. Many of these been seen by their own general practitioners in other countries. The other possible explanation was that some of these illness-related reattendances are actually social problems in disguise. There were a number of regular patients who presented to us each time with similar complaints and requested admission because no one could take care of them at home. In our study, doctor-related return visits were much lower than the figures reported by Pierce. ${ }^{1}$ Kelly ${ }^{6}$ and $\mathrm{Hu}^{7}$ also found a doctor error rate which was lower than those reported in North America. Part of the reason might be attributed to the difficulty encountered in ascertaining inadequate treatment or diagnosis in a retrospective study of patient charts.

Return visit patients were often considered a high risk group. The admission rate for this group was $36 \%$ in our study which is higher than the usual $25 \%$ admission rate for first attendance. Part of this could, however, be attributed to the fact that this might be a self-fulfilling prophecy. Most doctors were taught to treat patients making a return visit with greater respect and the threshold for admission is lower.

These results might be more meaningful if we could find out which groups are most likely to make a return visit. In terms of demographics we could see clearly that the young are the most vulnerable group (Fig. 1). The usual reason for reattendance was fever and about $40 \%$ of patients were admitted after the second visit. Further analysis of these patients might help us delineate characteristics of this group. It was surprising to find that the return visit rate of patients over 60 years of age was actually less than average. This could be because we had a lower threshold for admission for this group of patients in the first visit. In an admission analysis carried out in the first week of December 1991 , the admission rate of the over 60 years of age group was $51 \%$ which was significantly higher than the average admission rate.

Patients presenting with asthma or COAD were 
Reattendance audit also a high risk group. More than half of these reattenders were admitted. Many studies have been reported on predicting reattendance levels. However, so far, there seems to be no measures which are sensitive and specific enough. One study ${ }^{8}$ pointed out that social and psychological factors might play a significant role.

Patients who left against medical advice or who disappeared after initial treatment were also considered to be high risk and about $40 \%$ in this group were admitted on reattendance. Proper counselling of patients at the first visit would be valuable.

Abdominal pain was a common presenting symptom in EDs and about a third of patients reattending with these symptoms were admitted. One way to reduce unscheduled visits of this kind would be to offer next day follow-up to patients with abdominal pain if the pain had not subsided. Longer observation time with repeated examination in an observation ward might also help to decrease unscheduled reattendances.

The next question was how to define the group of patients whose return visit could be avoided if they were managed properly on the first visit. The rate of avoidable reattendances varied widely as reported in the literature. $\mathrm{Hu},{ }^{7}$ in Taiwan, reported a rate $(8 \%)$ which was much lower than the figure (over $30 \%$ ) reported by North American authors. ${ }^{3,4}$ Our rate was around $18 \%$ which was higher than that reported by $\mathrm{Hu}$. This variation could be explained in part by the fact that the judgements were retrospective and subjective. It would also depend on how complete the documentation was. Different criteria were used and thus strict comparison might not be meaningful. We defined avoidable reattendance as those return visits that were patient- or doctor-related. This could only be a crude measure as not all doctor-related reattendances were avoidable, e.g. an appendicitis might not have been detected at an early stage no matter how thorough the doctor was.

Another important question was how to do things right the first time to decrease avoidable reattendance. There were no uniform answers in the literature. It was suggested that better patient education could decrease the rate of return visits. $\mathrm{Hu}^{7}$ went a step further to conclude that more careful examination and interpretation of white cell count and chest radiographs could decrease some of the avoidable reattendances. The costeffectiveness aspects, however, were not studied. We would concur that in cases where patient-related factors were important, better education and instructions could prevent some return visits. But, in our setting, a better organized primary health care system with an effective interface with the hospital system seems to offer a better solution for the majority of illness related 'reattendances'. Alternatively, a better follow-up service at the ED would be necessary to decrease these unscheduled return visits. This option, however, may not be cost effective. $\mathrm{Hu}^{7}$ also suggested that return visits should be studied with respect to the seniority of the doctor. This was an interesting suggestion and it would not be too difficult to undertake with a computerized database containing all the pertinent information.

\section{CONCLUSIONS}

Audit of return visits is suggested as one activity which should be included in an ED quality assurance programme. Although the level of management errors that was detected might not be high, we found it useful as part of the quality assurance programme. It is not too time consuming to carry out audit on a daily basis if the patient database is computerized. It provided us with an early opportunity to correct any cases of mismanagement and alerted us to those patients with a high chance of making an unscheduled reattendance.

\section{REFERENCES}

1. Pierce J.M. (1990) "Bounces": An analysis of shortterm return visits to a public hospital emergency department. Annals of Emergency Medicine 19, 752-757.

2. Stair T.O. (1987) Quality assurance. Emergency medicine Clinics of North America 5(1), 41-50.

3. Lerman B. \& Kobernick M.S. (1987) Return visits to the emergency departments. Journal of Emergency Medicine 5(5), 359-362.

4. Keith K.D., Bocka, J.J., Kobernick M.S., Krome R.L. \& Ross M.A. (1989) Emergency department revisits. Annals of Emergency Medicine 18, 964-968.

5. Leicester M.C., Johnston J., Daniel L., Ng N.Y., Hedley A.J., Fung $H$. et al. (1990) HK Accident \& Emergency Departments: operational studies and medical audit on the demand for care, admission to hospital and clinical outcomes. Report of Hospital Services Department

6. Kelly A.M., Chirnside A.M. \& Curry C.H. (1992) Why do patients bounce? (abstract) Emergency Medicine 4, 199-254.

7. Hu S.C. (1991) Analysis of patients revisits to the emergency department. American Journal of Emergency Medicine 10, 366-370.

8. Stehr D.E., Klein B.J. \& Murata G.H. (1991) Emergency department return visits in chronic obstructive pulmonary disease: the importance of psychosocial factors. Annals of Emergency Medicine 20, 1113-1116. 